\title{
Shadow Inspection of 3D Objects in Partially Coherent Light
}

\author{
E.S. Senchenko ${ }^{1,2}$, Yu.V. Chugui ${ }^{1,3}$ \\ ${ }^{1}$ Technological Design Institute of Scientific Instrument Engineering SB RAS, 41, Russkaya St., 630058, Novosibirsk, \\ Russian Federation, chugui@tdisie.nsc.ru \\ ${ }^{2}$ Novosibirsk State Technical University, 20, K. Marksa Pr., 630092, Novosibirsk, Russian Federation \\ ${ }^{3}$ Novosibirsk State University, 2, Pirogova St., 630090, Novosibirsk, Russian Federation
}

The peculiarities of 3D object image formation in a diffraction-limited system using quasi-monochromatic source with finite angular size are investigated. Algorithms for considerable increase in the accuracy of inspection of 3D objects by shadow optical method are proposed. These algorithms are based on high precision analytical determination of shift of shadow boundary image of thick object and on introduction of corrections depending on the object thickness and the optical system parameters. The theoretical results obtained have been experimentally verified.

Keywords: 3D image formation, shadow method, dimensional inspection, partially coherent source

\section{INTRODUCTION}

$\mathrm{T}$ HE SHADOW systems, due to their high precision and operation speed as well as broad measurement range, are widely used among the optical means of noncontact dimensional inspection in industry [1]. The essence of the measurement shadow method consists in the position finding of the object's true boundary. It allows us to determine its different geometrical dimensions, including width, length, hole diameter, etc. In case of flat objects (zero thickness) the true boundary is determined using shadow image thresholding, either at $25 \%$ of light intensity illuminating the object (coherent illumination) or at $50 \%$ of this intensity (noncoherent illumination) [2]. Under inspection of 3D (thick) objects in coherent light the boundary position shift takes place $[3,4]$, which is proportional to the Fresnel zone.

Because of known difficulties occurring under the use of coherent illumination (first of all, speckle noises [5]), more promising is the partially coherent illumination for inspected 3D objects using, for instance, LED. Such partially coherent illumination was effectively used by us for a measurement system using Fresnel images of the inspected objects [6].

The peculiarities of $3 \mathrm{D}$ object image formation in a diffraction-limited system under their illumination by partially coherent light are examined. The methods for analytical determination of shift of 3D object image shadow boundary and its correction depending on the object thickness and the optical system parameters are proposed. These algorithms allow us to considerably increase the measurement precision of $3 \mathrm{D}$ objects by the shadow method.

\section{SHADOW MEASUREMENTS FOR 3D OBJECTS IN PARTIALLY COHERENT LIGHT}

The optical scheme of the shadow system for inspection of $3 \mathrm{D}$ objects is presented in Fig.1. Extended source 1 with angular sizes $2 \theta_{s}$ is illuminating a thick edge with width $\mathrm{d}$ through objective of lens 2. Projection lenses 4 and 6 are forming its shadow image on the photodetector matrix 7 .
Aperture diaphragm 5 with angular sizes $2 \theta_{a p}$ is located at focal distance from the lens 4 .

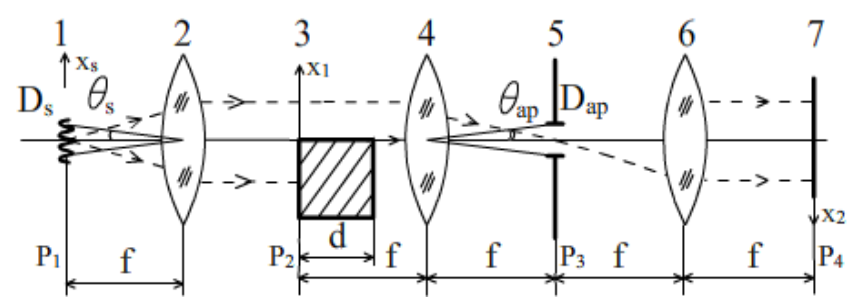

Fig.1. Optical scheme for formation of 3D object image in a diffraction-limited system.

The image intensity $I(x 2)$ profile of the $3 \mathrm{D}$ object's edge can be seen in Fig.2.

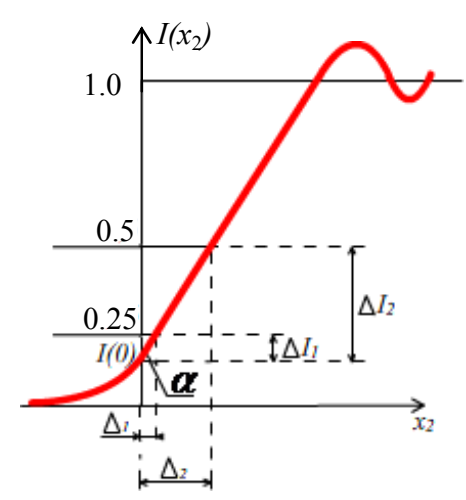

Fig.2. Image profile intensity $I(x 2)$ of the 3D object's edge on the photodetector matrix $(I(x))$ is normalized intensity).

The value of threshold $I_{t h r}=I(0)$ depends on some parameters, including angular sizes of the light source and the aperture diaphragm, as well as the object's volumetricity. For flat objects $(\mathrm{d}=0)$ illuminated by coherent light the threshold is equal to $I_{t h r}^{(1)}=0.25\left(\theta_{s} \rightarrow 0 \mathrm{rad}\right)$, and in case of noncoherent light illumination the threshold 
equals $I_{t h r}^{(2)}=0.5\left(\theta_{s} \rightarrow \pi / 2 \mathrm{rad}\right)$. Under partially coherent illumination the threshold value is intermediate within the limits of $0.25<I_{t h r}<0.5\left(0 \mathrm{rad}<\theta_{s}<\pi / 2 \mathrm{rad}\right)$ [2].

The influence of $3 \mathrm{D}$ object's thickness on its image profile is determined by the ratio of critical diffraction angle $\theta_{c r}=\sqrt{\lambda / d} \quad$ (under which the volume effects become significant) to the angular size of the aperture $2 \theta_{a p}[3,4]$ ( $\lambda$ is wavelength). If at $\theta_{c r}>>\theta_{a p}$ the weak volume effects occur, so at $\theta_{c r} \ll<\theta_{a p}$ their effects are sufficiently significant. Further calculations are limited by weak volume effects taking place in practical applications.

\section{ALGORITHMS FOR HIGH PRECISION DETERMINATION OF 3D OBJECT BOUNDARIES}

Two algorithms to determine the position of the geometric boundary of thick edge (plane perfectly absorption surface parallel to the optical axis), have been developed. The first one is based on the use of threshold $I_{t h r}=I(0)$ (Fig.1) that takes into account the angular size of the source $2 \theta_{s}$ and object's thickness d. For calculation we used the constructive theory of image formation for the thick objects with the sharp shadow projections [3]. It has been shown that the normalized light intensity in the thick edge image in point $x_{2}=0$ (that coincides with boundary geometric position) under $\theta_{s} \ll \theta_{a p}$ is equal to (see appendix A):

$$
I_{t h r}=I(0)=\frac{1}{4}+\frac{1}{3 \pi^{2}} \frac{\theta_{s}^{2}}{\theta_{a p}^{2}}-\frac{\theta_{a p}}{\sqrt{2} \pi \theta_{c r}}
$$

Thus, value $I(0)$ is determined by the three components. The first term of the equation corresponds to the case when a flat object is illuminated with point axial light source (coherent illumination). The second one refers to the influence of the final angular size of source and results in the increase of the threshold level. Finally, the third component is determined by the object volumetricity, which decreases the value of $I(0)$. Thus, at given parameters of $\mathrm{d}$ and $\theta_{a p}$ there is an opportunity, by choosing the angular size of the source $2 \theta_{s}$, to compensate the effect of the object's volumetricity on the change of light intensity at the point of geometric position of the thick edge boundary $\left(x_{2}=0\right)$. It is possible due to different signs of the second and third terms in (1).

Computer modeling has allowed obtaining the dependency of the threshold level on the light source diameter (Fig.3). According to the obtained results (1) the threshold levels for the thick and flat edges illuminated by light source of different size are differed by the third component. Under $\theta_{s}=\theta_{a p}$, threshold level jump is observed. The theoretical results as well as computer modeling ones are in good agreement.

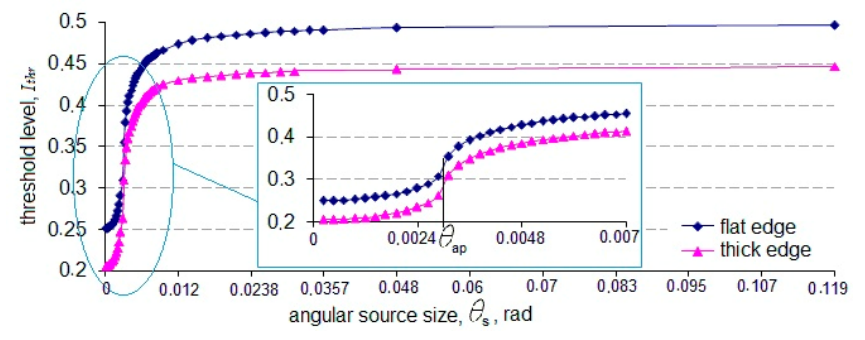

Fig.3. The threshold level $I_{t h r}$ for shadow image vs. the light source size $\theta_{s}$.

The second algorithm developed represents the determination of the position of the 3D edge's boundary using the two standard thresholds: $I_{t h r}^{(1)}=0.25$ and $I_{t h r}^{(2)}=0.5$. It is important that the high precision determination of the boundaries requires introduction of two corrections $\Delta_{1}$ and $\Delta_{2}$ that are determined by the following equations (Fig.2):

$$
\Delta_{1}=\frac{0.25-I(0)}{I^{\prime}(0)}=\frac{\Delta I_{1}}{\operatorname{tg} \alpha}, \quad \Delta_{2}=\frac{0.5-I(0)}{I^{\prime}(0)}=\frac{\Delta I_{2}}{\operatorname{tg} \alpha},
$$

where $\operatorname{tg} \alpha=I^{\prime}(0)$ is the slope angle of the 3D edge's image profile under the selected level. In this case, the value of $I^{\prime}(0)$ has to be known. For finding this value it is necessary to determine a derivative of $I(x 2)$ in a point $\mathrm{x}=0$. It's established that at $\theta_{s}<<\theta_{a p}$ (see appendix B):

$$
I^{\prime}(0)=\frac{2 \theta_{a p}}{\lambda}+\frac{4}{\sqrt{2} \pi \lambda \theta_{c r}}\left(\frac{1}{3} \theta_{s}^{2}-\theta_{a p}^{2}\right)
$$

Taking into account (2), (3), for corrections $\Delta_{1}$ and $\Delta_{2}$ one can obtain the following:

$$
\begin{aligned}
& \Delta_{1}=\frac{\lambda}{2 \theta_{a p}}\left[\frac{1}{\sqrt{2} \pi} \frac{\theta_{a p}}{\theta_{c r}}-\frac{1}{3 \pi^{2}} \frac{\theta_{s}^{2}}{\theta_{a p}^{2}}\right] \\
& \Delta_{2}=\frac{\lambda}{2 \theta_{a p}}\left[0.25+\frac{3}{2 \sqrt{2} \pi} \frac{\theta_{a p}}{\theta_{c r}}-\frac{1}{3 \pi^{2}} \frac{\theta_{s}^{2}}{\theta_{a p}^{2}}\right]
\end{aligned}
$$

According to (4), the values of the corrections depend on the critical angle, the angular sizes of the aperture and the light source. The corrections can be minimized through choice of the parameter system due to the different signs of the components in (4).

\section{EXPERIMENTAL RESULTS AND DISCUSSION}

The theoretical results obtained have been experimentally verified. The scheme of the experimental optical system is presented in Fig.1. As light source 1, LED with effective wavelength $\lambda=628 \mathrm{~nm}$ and spectral width $\Delta \lambda=15 \mathrm{~nm}$ was used. Necessary angular size of the light source was set using a diaphragm. The homogeneity illumination of the 
inspected object was reached by mat diffuser. The thick object 3 was projected by lenses 4 and 6 on a photodetector camera with $2210 \times 3002$ pixel matrix (pixel size was $3.5 \mu \mathrm{m}$ ). The angular size of aperture diaphragm 5 had been set on the assumption that $\theta_{a p}>\theta_{s}$. The system calibration was fulfilled by using a thin object with the thickness of a few microns.

In experiments we used ceramic isolation rings with thickness within $0.8-80 \mathrm{~mm}$, with outer diameter of $28-$ $80 \mathrm{~mm}$ and inner diameter of $23-62 \mathrm{~mm}$ as the thick objects. The position of thick edge was determined at level $I_{t h r}^{(2)}=0.5$. By computer processing of the obtained shadow images from the photodetector camera it is possible to determine the boundary position of 3D objects with an improved accuracy up to the tenth part of a pixel size.

The influence of the object's thickness and of the optical system parameters on the image position profile has been experimentally established and theoretically confirmed. For example, when the measuring thickness of the threedimensional edge varied from 2 to $10 \mathrm{~mm}$, the position boundary shift was equal to $4.77 \mu \mathrm{m}$. The results of the experiment and computer modeling are presented in Fig.4, which shows the dependence of ring edge's shift on the thickness. One can see that the deviation of the experimental and theoretical data in average has not exceeded a few microns that can be explained by non accuracy position under calibration of the initial objects (instrument error was about $2 \mu \mathrm{m}$ )

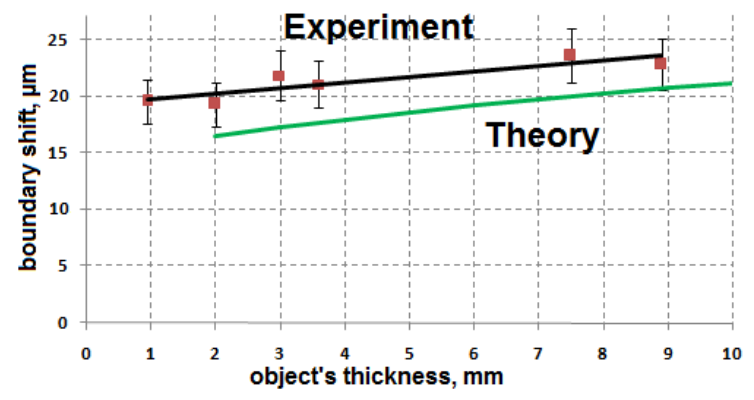

Fig.4. Shift of the position of 3D object's edge vs. the object's thickness: points are experimental data; solid line is averaged experimental curve; the curve below is calculation data.

Using the second algorithm we have been able to decrease the systematic error of the inspection system ten times: from $20 \mu \mathrm{m}$ to $2 \mu \mathrm{m}$.

\section{CONCLUSION}

We have studied the peculiarities of image formation for $3 \mathrm{D}$ objects, as a thick edge with sharp shadow projection illuminated by partially coherent light applied to dimensional inspection. The research results provide evidence that shift of 3D object shadow boundary depends on the object's thickness, the angular sizes of the light source and the aperture. It is shown that the choice of source size can minimize the shift of real $3 \mathrm{D}$ edge position. The developed algorithms for processing the measurement information allow a significant increase in the precision of
3D object geometrical parameter measurement by choosing the angular size of light source. The results obtained can be used for development of precision measurement systems for 3D object inspection with sharp shadow projection.

\section{APPENDIX A}

Let's obtain the derivation of $I\left(x_{2}\right)$ under $\theta_{s}<<\theta_{a p}$. As it is known [3], the spectrum of perfectly absorbing thick edge (Fig.1) under illumination by normal plane wave (formed by an axis point source) is defined:

$$
F(\omega)=\pi \delta(\omega)+\frac{\tilde{Y}_{d}(\omega)}{i \omega}+\frac{Y(0)}{i \omega} e^{i \omega^{2} d} \frac{}{2 k}
$$

where $\omega=k \theta$ is angular spatial frequency; $\theta$ is diffraction angle; $k=2 \pi / \lambda$ is wave number ( $\lambda$ is wavelength); $\delta(x)$ is Dirac delta function; $\widetilde{Y}_{d}(\omega)$ is the Fresnel function [7]; $d$ is object's thickness; $Y(x)$ is Heaviside step function.

The Fresnel function in case of weak object's volumetricity, i.e. at $\omega_{a p}=k \theta_{a p}<<\omega_{c r}=k \theta_{c r}$ can be written [4]:

$$
\widetilde{Y}_{d}(\omega)=0.5+e^{-i \pi / 4} \frac{\omega}{\omega_{c r}}
$$

where $\omega_{c r}=k \theta_{c r}=k \sqrt{\lambda / d}=2 \pi / \sqrt{\lambda d}$ is angular critical spatial frequency.

Taking into account (A2) and $e^{\frac{i \omega^{2} d}{2 k}} \rightarrow 1$, (A1) can be transformed:

$$
F(\omega)=\left[\pi \delta(\omega)+\frac{1}{i \omega}\right]+\frac{e^{-i \pi / 4} \frac{\omega}{\omega_{c r}}}{i \omega}=\left[\pi \delta(\omega)+\frac{1}{i \omega}\right]+\frac{e^{-i \pi / 4}}{i \omega_{c r}}
$$

In case of illumination of $3 \mathrm{D}$ object by inclined plane wave (formed by point off-axis source shifted on value $\omega_{s h}$ ) its spectrum can be written:

$$
F\left(\omega, \omega_{s h}\right)=\pi \delta\left(\omega-\omega_{s h}\right)+\frac{1}{i\left(\omega-\omega_{s h}\right)}-\frac{e^{i \frac{\pi}{4}}\left(\omega-2 \omega_{s h}\right)}{\left(\omega-\omega_{s h}\right) \omega_{c r}} .
$$

The amplitude field from any shifted point source in image plane is determined by the integral of thick edge spectrums (A3) within aperture diaphragm $2 \omega_{a p}$ :

$$
A\left(x_{2}, \omega_{s h}\right)=\frac{1}{2 \pi} \int_{-\omega_{a p}}^{\omega_{a p}} F\left(\omega, \omega_{s h}\right) \cdot \exp \left(i \omega x_{2}\right) d \omega .
$$

Resultant intensity of partially coherent illumination is the 
integral of square of amplitudes from each separate point source within source size $2 \omega_{s}$ :

$I\left(x_{2}\right)=\frac{1}{4 \pi^{2}} \int_{-\omega_{s}}^{\omega_{s}}\left|\int_{-\omega_{a p}}^{\omega_{a p}} F\left(\omega, \omega_{s h}\right) \times \exp \left(i \omega x_{2}\right) d \omega\right|^{2} d \omega_{s h}$.

In the vicinity of $3 \mathrm{D}$ edge boundary $\left(x_{2}=0\right)$ resultant intensity is equal to:

$$
I(0)=\frac{1}{4 \pi^{2}} \int_{-\omega_{s}}^{\omega_{s}}\left|\int_{-\omega_{a p}}^{\omega_{a p}} F\left(\omega, \omega_{s h}\right) d \omega\right|^{2} d \omega_{s h} .
$$

After some calculations, the following equation can be obtained:

$$
I(0)=\frac{1}{4}+\frac{1}{3 \pi^{2}} \frac{\theta_{s}^{2}}{\theta_{a p}^{2}}-\frac{\theta_{a p}}{\sqrt{2} \pi \theta_{c r}} .
$$

\section{APPENDIX B}

For the calculation of image intensity profile derivative for 3D object we will use the spectral representations (Fig.1). The intensity derivative is defined (see appendix A):

$$
I^{\prime}\left(x_{2}\right)=\left(\int_{-\omega_{s}}^{\omega_{s}} A\left(x_{2}, \omega_{s h}\right) A^{*}\left(x_{2}, \omega_{s h}\right) d \omega_{s h}\right)^{\prime}
$$

where asterisk denotes the complex conjugate.

Then for $I^{\prime}(0)$ at the vicinity of $3 \mathrm{D}$ edge boundary $\left(x_{2}=0\right)$ we can receive the following:

$$
\begin{aligned}
& I^{\prime}(0)=\int_{-\omega_{s}}^{\omega_{s}} A^{\prime}\left(0, \omega_{s h}\right) A^{*}\left(0, \omega_{s h}\right) d \omega_{s h}+ \\
& +\int_{-\omega_{s}}^{\omega_{s}} A\left(0, \omega_{s h}\right)\left(A^{*}\left(0, \omega_{s h}\right)\right)^{\prime} d \omega_{s h}
\end{aligned}
$$

Taking into account (A4), amplitude derivative is defined:

$$
A^{\prime}\left(x_{2}, \omega_{s h}\right)=\frac{1}{2 \pi} \int_{-\omega_{a p}}^{\omega_{a p}} F\left(\omega, \omega_{s h}\right) \cdot i \omega \cdot \exp \left(i \omega x_{2}\right) d \omega .
$$

According to (A3), (B2), at the vicinity of 3D edge boundary $\left(x_{2}=0\right)$ one can obtain:

$$
\begin{aligned}
& A^{\prime}\left(0, \omega_{s h}\right)=\frac{1}{2 \pi} \int_{-\omega_{a p}}^{\omega_{a p}} F\left(\omega, \omega_{s h}\right) \times i \omega d \omega= \\
& =\frac{1}{2 \pi} \int_{-\omega_{a p}}^{\omega_{a p}}\left[\pi \delta\left(\omega-\omega_{s h}\right) i \omega+\frac{\omega}{\left(\omega-\omega_{s h}\right)}-\frac{i \omega \times e^{i \frac{\pi}{4}}\left(\omega-2 \omega_{s h}\right)}{\left(\omega-\omega_{s h}\right) \omega_{c r}}\right] d \omega
\end{aligned}
$$

Turning back to (B1) after some transformations, the final expression for an image profile slot of the $3 \mathrm{D}$ object in the field of its border can be obtained as:

$$
I^{\prime}(0)=\frac{2 \theta_{a p}}{\lambda}+\frac{4}{\sqrt{2} \pi \lambda \theta_{c r}}\left(\frac{1}{3} \theta_{s}^{2}-\theta_{a p}^{2}\right) .
$$

\section{REFERENCES}

[1] Webster, J.G. (1999). The Measurement, Instrumentation, and Sensors Handbook. Boca Raton: CRC Press LLC.

[2] Goodman, J.W. (1968). Introduction to Fourier Optics. New York: McGraw-Hill.

[3] Chugui, Yu.V., Krivenkov, B.E. (1989). Fraunhofer diffraction by bodies of constant thickness. JOSA, A 6 , 617-626.

[4] Chugui, Yu.V. (2001). Optical dimensional metrology for 3D objects of constant thickness. Measurement, 30, 19-31.

[5] Goodman, J.W. (2006). Speckle Phenomena in Optics: Theory and Applications. Englewood, CO: Roberts \& Company Publishers.

[6] Chugui, Yu.V., Yakovenko, N.A., Yaluplin, M.D. (2006). Metrology for Fresnel measuring method. Meas. Sci. Technol., 17, 592-595.

[7] Papoulis, A. (1968). Systems and Transforms with Applications in Optics. New York: McGraw-Hill.

Received June 15, 2011. Accepted September 9, 2011. 